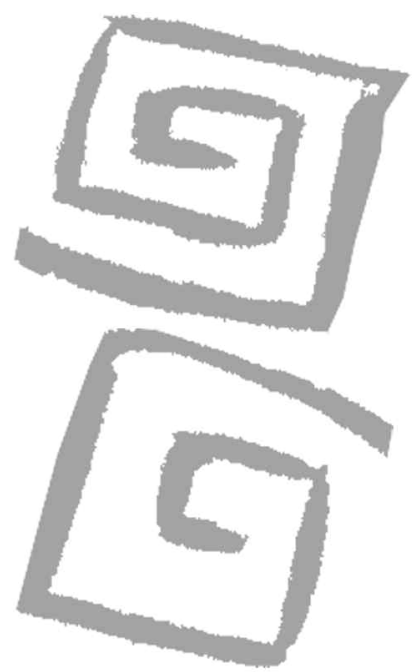

\title{
La enfermedad en su laberinto: avances, desafíos y paradojas de cien años del Chagas en Argentina
}

\author{
The disease in its labyrinth: advances, challenges and \\ paradoxes over 100 years of Chagas in Argentina
}

${ }^{1}$ Doctor en Ciencias Sociales, Facultad Latinoamericana de Ciencias Sociales. Doctor en Historia de la Ciencia, Université Paris 1 Panthéon Sorbonne. Investigador CONICET, Instituto de Estudios sobre la Ciencia y la Tecnología, Universidad Nacional de Quilmes. Director, Archivo General de la Nación Argentina. jpzeta@unq.edu.ar

Zabala, Juan Pablo'

RESUMEN El artículo analiza la persistencia de la enfermedad de Chagas en la Argentina durante más de un siglo, prestando atención a las diferentes dimensiones (biológica, de conocimiento, política, profesional y técnica) que participan en su definición. Se pone el acento en la identificación y discusión de algunas de las tensiones fundamentales que han marcado la historia de la enfermedad, con la intención de discutir cuáles han sido los condicionamientos concretos que han marcado, por un lado, la continuidad de la enfermedad dentro de la consideración política pero, al mismo tiempo, la imposibilidad de alcanzar una situación de control definitivo.

PALABRAS CLAVE Enfermedad de Chagas; Conocimiento; Historia; Políticas.

ABSTRACT This article analyzes the century-long persistence of Chagas disease in Argentina, taking into account the different dimensions (the biological, the informational, the political, the professional and the technical) that take part in the disease's definition. Emphasis is placed on the identification and discussion of fundamental tensions that have marked the history of the disease, with the intention of discussing the concrete conditioning factors that have, on the one hand, permitted the continuity of Chagas as a subject of political consideration, but on the other have made it impossible to reach a situation of definitive control over the disease.

KEY WORDS Chagas Disease; Knowledge; History; Policies. 


\section{INTRODUCCIÓN}

La enfermedad de Chagas, o tripanosomiasis americana, cumplió en 2009 un siglo de existencia, desde que Carlos Chagas anunciara el descubrimiento del Trypanosoma cruzi (el parásito causante), el insecto vector (el barbeiro o vinchuca en Argentina) y un conjunto de síntomas clínicos característicos, en el Brasil de principios de siglo XX donde el control de las enfermedades infecciosas parecía un boleto seguro al orden y el progreso $(1,2)$.

A lo largo del siglo $\mathrm{XX}$, la enfermedad de Chagas se convirtió en un símbolo de las enormes porciones de la población que en América Latina viven en condiciones de pobreza material, especialmente de las zonas rurales, con escasez de información y falta de acceso a servicios sanitarios básicos. Actualmente, esta situación se traduce en cifras oficiales que reconocen ocho millones de personas infectadas con el parásito, susceptibles de padecer las dolencias crónicas cardíacas y digestivas que caracterizan a la enfermedad, y otras 100 millones en riesgo de infectarse; 56.000 nuevos casos anuales y 12.000 muertes producidas por año (3).

Desde hace más de medio siglo, el Chagas ha sido reconocido como un problema sanitario de primer orden, al menos discursivamente, tanto en Argentina como en el resto de América Latina, y se han desarrollado distintos programas de intervención orientados a su control. Estos planes han incluido diversas acciones, tales como la eliminación de los vectores, la promoción de la investigación en distintas áreas de conocimiento, la regulación de las transfusiones sanguíneas, la realización de campañas educativas, el mejoramiento de viviendas. Al mismo tiempo, desde mediados de la década de 1970 están disponibles los actuales tratamientos antiparasitarios, a los que se suman alternativas de tratamiento de los síntomas cardíacos o digestivos mediante alternativas no específicas de las afecciones chagásicas.

¿Cómo entender la persistencia de la enfermedad durante todas estas décadas, en un contexto en el que se encuentran disponibles los medios técnicos para su control, y la decisión política de destinar recursos para su combate?
En este artículo intento responder a esta pregunta a partir de la idea de que la enfermedad de Chagas está atravesada por una tensión fundamental entre el reconocimiento y el olvido que ha marcado su historia. Es decir, por la existencia de acciones que han destacado la relevancia del Chagas como problema social, posicionándolo en el ámbito de las políticas y de la conciencia colectiva como un asunto sobre el que es necesario intervenir, junto con otras que han tendido a invisibilizar el problema, tanto en el plano político, médico como simbólico. El resultado es un conjunto de dilatadas iniciativas orientadas al control de la enfermedad por diferentes vías, pero también la imposibilidad de llegar a ese objetivo en forma definitiva. En otras palabras, la enfermedad de Chagas no ha sido ni tan olvidada como para desaparecer de la agenda, ni tan recordada como para cortar definitivamente su ciclo de reproducción.

Es necesario explorar esa tensión, analizar la forma en que ella se inscribe en las racionalidades, creencias e intereses de los distintos actores involucrados en la definición de la enfermedad, tanto de los políticos, médicos, investigadores, infectados y enfermos, para poder escapar a dos respuestas típicas para explicar el "fracaso" en el control del Chagas. La primera de estas posturas es eminentemente cientificista y tecnocrática, y pone el énfasis en la falta de desarrollo de los medios adecuados para el control, por ejemplo, de una vacuna, una droga antiparasitaria más eficiente, o un insecticida de mayor acción residual. Desde esta perspectiva, es el avance de la ciencia y la tecnología el que permitirá, eventualmente, poder dar fin al problema (a). El segundo tipo de explicación, en cambio, suele ser meramente política-comercial: la persistencia del Chagas tiene que ver con la falta de interés político, por tratarse de una enfermedad de pobres $-y$ por ende de sujetos no capaces de generar beneficios/ganancias-, y la única manera de solucionar el problema es definir las prioridades políticas con criterios no mercantilistas.

Cuando miramos de cerca a la enfermedad, vemos que la realidad no responde a estos esquemas simplistas. El Chagas es una entidad compleja, condicionada tanto por los procesos biológicos involucrados como por los intereses profesionales, económicos, políticos, culturales e institucionales que se ponen en juego en 
la definición de lo que la enfermedad es, y sobre todo, de la forma en la que debe intervenirse sobre ella. Como consecuencia de esta complejidad, la trayectoria de la enfermedad de Chagas encierra una serie de paradojas que han marcado su dilatada historia:

a) Un hito en la historia de la ciencia, y una entidad que tiende a hacerse invisible en la actual atención médica.

b) Una "enfermedad olvidada", y una constante en las agendas de las políticas públicas.

c) Un relativo éxito en términos de combate del vector, y la persistencia de una realidad que favorece su desarrollo.

d) La mayor población afectada, y el grupo social menos visible en términos de identificación y organización social.

e) Una enfermedad rural, pero un problema sanitario de zonas urbanas y una amenaza para países desarrollados.

f) Una bandera importante del compromiso de la comunidad científica con la realidad social, y una marca de la escasa incidencia concreta de la ciencia en las condiciones de vida de las personas.

g) Un área de conocimiento con un alto grado de avance, y medicamentos de alrededor de 40 años de antigüedad y limitada efectividad.

Analizaremos estas paradojas a partir de tres núcleos fundamentales de definición del Chagas, que nos permitan entender, en la especificidad de la enfermedad (5), la manera en que se ha expresado históricamente la tensión entre visibilidad e invisibilidad, siempre como consecuencia de un mutuo condicionamiento entre las diferentes dimensiones técnicas, biológicas, de conocimiento, profesionales y políticas. El primero de estos nodos refiere a la definición de la enfermedad en sus aspectos biomédicos, tanto de su caracterización como de la administración de los tratamientos. El segundo, a la manera en que la enfermedad se constituyó en un objeto de políticas públicas de distintos órdenes, con la consiguiente aplicación de diferentes planes de intervención. Y el tercero, a la enfermedad como un marco estructurante de identidades y relaciones, que condicionan y preforman la identidad de los sujetos afectados.

\section{DESCUBRIMIENTO, REDEFINICIÓN Y DESAPARICIÓN DE LA ENFERMEDAD}

Hay un punto en el que las enfermedades se diferencian del resto de los problemas sociales, y es que su definición toma siempre como referencia a uno o varios eventos biológicos que, a través de las miradas expertas del conocimiento científico y profesional, condicionan cuál es la manera en que la enfermedad es concebida. Ello nos obliga a considerar que la propia definición de esos hechos -en apariencia objetivos- es también producto de procesos sociales en los que también es posible encontrar marcas que condicionan la forma en que la enfermedad finalmente se constituye como problema social.

En el caso del Chagas, el proceso de constitución de la enfermedad como una entidad médica estable es particularmente rico para explorar la tensión entre visibilidad e invisibilidad, pues las profundas modificaciones en el plano cognitivo que ha tenido a lo largo de su historia han hecho inestable la demarcación del problema. La enfermedad de Chagas tuvo una aparición rutilante en el mundo médico y científico de principios del siglo XX. El hecho es reconocido como caso excepcional por la historia de la ciencia y la medicina tradicionales, pues en un mismo acto, en un período de tiempo muy corto, una sola persona fue capaz de descubrir todos los componentes de una nueva enfermedad infecciosa: el agente causal (el Trypanosoma cruzi), el vector (la vinchuca o barbeiro) y el cuadro clínico de la enfermedad.

En un primer momento, la caracterización de la enfermedad hecha por Chagas en sus trabajos, afirmaba que la etapa aguda, cuando la infección es reciente, "resulta, casi siempre, en la muerte [...], sobre todo en niños menores de 6 meses", aunque en los niños "de más de un año, no siempre el resultado es fatal; muchos de los enfermos consiguen pasar a la fase crónica" (6 p.263). Pero sin dudas, fue la fase crónica (b), referida a los efectos de la infección en el largo plazo, la que mayor interés despertó en la comunidad médica y en los encargados de la política sanitaria. En esta fase de la enfermedad, afirmaba Chagas, el parásito producía serias perturbaciones funcionales de la glándula tiroides y del sistema 
nervioso, de modo tal que se proponía como una explicación a la salud de una región caracterizada por altos índices de bocio; extensos casos de anemia profunda con gran decaimiento orgánico, lo que provocaba retraso de la evolución en los niños e infantilismo en los adultos; deficiencias notables del sistema nervioso y, asociado a ello, discapacidades para el habla y la comunicación, y donde además abundaban los casos de imbecilidad (7). A ello agregaba Chagas que "en algunos casos [...], especialmente en adultos, son muy frecuentes las perturbaciones del ritmo cardíaco" (6 p.265).

La resonancia que tuvo el anuncio de Chagas no residió solamente en la habilidad científica para la identificación de una nueva especie, sino en la capacidad de esas afirmaciones de proponer una explicación de base biológica a una situación social de carencia, atraso y falta de desarrollo, a través de la identificación de causas invisibles al común de las personas. A partir de esa demarcación, los ojos experimentados de un científico formado en la frontera de la protozoología de la época, con acceso a un laboratorio científico y con un prestigio cimentado en anteriores campañas sanitarias exitosas (c), ofrecían una nueva lente para entender el malogrado devenir de décadas y décadas de millones de personas diseminadas en la inmensa región del Brasil alejado de las costas. $Y$ a partir de allí, articular un movimiento político -liderado en buena medida por Chagas- que proponía un "saneamiento del Brasil" a través de la erradicación de ciertos agentes perniciosos para la salud pública, donde la investigación científica era una punta de lanza de estas medidas $(9,10)$.

Sin embargo, la descripción que realizó Chagas en los primeros años de la enfermedad fue luego objeto de largos debates profesionales que ponían en duda la gravedad del asunto, y marcaron el paso de una primera etapa de amplio reconocimiento a otra signada por la incerteza, las controversias y, en definitiva, el olvido. En particular, la dificultad para presentar los casos que confirmaran la extensión de la enfermedad (d) y el cuestionamiento a la relación entre el parásito y el bocio, significaron una puesta en jaque radical a los cimientos en los que se sustentaba el problema sanitario (e).
Muchos años pasaron, desde entonces, para llegar a la definición actual de la enfermedad, caracterizada por las afecciones cardíacas y digestivas. Desde principios de la década de 1920 hasta fines de la década siguiente, las investigaciones sobre la enfermedad apuntaron a la definición de cuáles eran efectivamente los efectos del parásito, cómo identificar a los infectados, y si podía distinguirse un cuadro clínico específico. En esa tarea cobraron especial importancia las investigaciones llevadas adelante por Salvador Mazza en la Misión de Estudios de Patología Regional del Norte a partir de mediados de la década de $1920(13,14)$. Mazza retomó, en una época en la que se creía que la enfermedad no existía en Argentina, el interés por el tema, en el contexto de un programa de estudio de patologías regionales del norte argentino, impulsando y articulando distintos estudios sobre el tema que retomaron varias de las preocupaciones iniciales de Chagas (f).

En ese marco, la importancia de la enfermedad aumentaba a medida que crecía la recopilación de "casos agudos", que se vio favorecida por la identificación del "Signo de Romaña" -inflamación del párpado como reacción ante la picadura de la vinchuca- que favoreció enormemente la difusión de la enfermedad entre los médicos no expertos $(2,9,16,17,18)$. Y hacia fines de la década de 1930, la enfermedad comenzaba a ser reconocida como un problema sanitario de envergadura por parte del campo científico-médico, sustentado en la acumulación de casos certificados y en las intensas actividades de divulgación de Mazza y sus colaboradores.

Sin embargo, pese al resurgimiento del Chagas como tema de interés profesional, aún era errática la definición del cuadro clínico de la enfermedad crónica. Por cierto, el bocio y las afecciones nerviosas fueron perdiendo espacio en la definición del cuadro clínico, y la forma cardíaca de la enfermedad se convirtió, desde la década de 1930, en un interés central de las investigaciones, tanto en Argentina como por parte del propio Chagas y sus discípulos, que mantenían, en un núcleo reducido, el interés por el tema en Brasil: "Discutíveis são as relações de causa e efeito, acaso existentes, entre o bócio e a infecção pelo Trypanosoma cruzi. [...] É uma 
questão aberta, a merecer estudo e perspicácia" (19 p.63). Pero fue recién a partir de la década de 1950 que la cardiopatía chagásica se constituyó en un objeto de indagación sistemática por parte de los cardiólogos, quienes definieron en el marco de esa especialidad tanto sus características específicas en relación con otras dolencias cardíacas como su forma de diagnóstico a través del electrocardiograma de la "miocardiopatía chagásica crónica"(g).

Con la descripción del cuadro cardíaco, la enfermedad rompió la barrera aleatoria que separaba a los infectados de los enfermos: estar enfermo de Chagas ya no era simplemente ser positivo a la prueba bioquímica de infección con el parásito, sino desarrollar una dolencia específica, reconocible con los instrumentos y saberes de la cardiología. Y, en consecuencia, su importancia como problema sanitario se empezó a sustentar en un número concreto de individuos que sufrían de una molestia inhabilitante para el trabajo, que se traducía en padecimiento para quienes la portaban y en pérdidas mensurables para la capacidad productiva del país por la disminución de su fuerza de trabajo.

Esto implicó una reconfiguración del significado de la enfermedad, en la medida en que emergió una especialidad médica específica, la cardiología, como el espacio de conocimiento y de práctica profesional legítimos para definir quiénes son los enfermos, cuáles los síntomas y cuál es la gravedad de los mismos. Y al mismo tiempo, una relocalización espacial del problema, en la medida en que los centros de atención pública de la ciudad de Buenos Aires comenzaron a diagnosticar casos de enfermos crónicos de Chagas entre los pacientes atendidos, migrantes de las zonas rurales que venían a trabajar a la ciudad.

A grandes rasgos, durante las últimas décadas la visibilidad de la enfermedad dentro del mundo médico se ha mantenido dentro de esta articulación de conocimientos y prácticas. Y esto ha tenido su correlato en el mundo profesional y en el seno de las instituciones de salud: una comunidad de cardiólogos especialistas en Chagas y la creación de servicios de Chagas en distintos hospitales son el reflejo visible de que el tema ha permanecido con cierta vigencia dentro del mundo de la sanidad.
Sin embargo, a esta visibilidad se oponen otras tendencias que atentan contra el reconocimiento del Chagas como una entidad mórbida autónoma. En particular, en los últimos años es posible observar al menos dos fenómenos que tienden a invisibilizar a la enfermedad. En primer lugar, el avance de la cardiología Ileva cada vez más a tratar la enfermedad, por parte de los mismos cardiólogos, por los síntomas coronarios de los pacientes, sin hacer distinción de que esas deficiencias tengan un origen en la infección parasitaria. Ya sea a través de drogas, marcapasos o microdesfibriladores, la miocardiopatía chagásica recibe el tratamiento específico que tiende a revertir esos síntomas, del mismo modo que cualquier afección cardíaca de otro origen. En otras palabras, para tratar a la enfermedad de Chagas no es necesario ser un especialista en el tema específico.

Este fenómeno tiene su correlato en el mundo de la organización profesional. Ya sea por falta de desafíos cognitivos y profesionales, ya sea por la escasa perspectiva económica que ofrece, el Chagas no aparece dentro de las carreras profesionales como una especialidad atractiva, y las comunidades de expertos mantienen a sus antiguos miembros, pero tienen una escasa capacidad de recambio generacional. En consecuencia, si bien puede ser reconocida dentro de los discursos sanitarios como un importante problema de salud, la enfermedad de Chagas tiende a invisibilizarse dentro de la actual práctica médica profesional, y queda diluida dentro de los procedimientos no específicos de su etiología.

Recién en los últimos años existen iniciativas que intentan recuperar la importancia de atender la etiología parasitaria de la dolencia cardíaca de origen chagásico, y proponen la aplicación del tratamiento antiparasitario en los enfermos crónicos. Por cierto, esta postura no ha estado exenta de polémicas y controversias (20), ya que existen al menos dos posturas contrapuestas al respecto sin que exista aún evidencia concluyente al respecto. Así, de un lado encontramos a quienes sostienen que una vez que la infección con el parásito ha sobrepasado una cierta cantidad de años, el tratamiento antiparasitario no es efectivo y no se justifica, ya que no produce beneficios y expone al paciente a los efectos secundarios perniciosos de la droga. Del otro 
lado, hay quienes sostienen que el tratamiento es beneficioso aun en los enfermos crónicos, aunque la demostración de la negativización de la infección solo se haga evidente en los análisis serológicos luego de varios años. En los últimos años, esta última postura ha ido ganando posiciones, y es oficialmente recomendado el tratamiento en niños y adolescentes. Pero más allá de la manera en que se resuelva finalmente la controversia, lo interesante es que detrás de la discusión científica se perciben dos maneras diferentes de delinear la enfermedad desde el punto de vista médico: una que atiende solamente a los enfermos que sufren dolencias cardíacas o gastrointestinales (que son solamente el $30 \%$ de los infectados), con tratamientos que son generales a dolencias similares con orígenes diversos, y otra que recupera la especificidad de la enfermedad, atacando su etiología, y proponiendo la intervención sobre el total de los infectados.

\section{EL CHAGAS COMO OBJETO DE POLÍTICA: ENTRE EL OLVIDO Y LA PRESENCIA}

El Chagas integra el grupo de las Ilamadas "enfermedades olvidadas". Esta denominación ha sido aplicada a un conjunto de enfermedades endémicas de gran extensión, que afectan principalmente a poblaciones de bajos recursos de América Latina, África y Asia, y que causan grandes pérdidas en términos de mortalidad y morbilidad. En particular, la denominación se aplica a aquellas enfermedades que no revisten un interés económico para la industria farmacéutica que justifique el desarrollo de nuevos fármacos, lo que tiene como consecuencia un escaso dinamismo en relación con el desarrollo de nuevos y más efectivos tratamientos, tal como sucede en el caso del Chagas.

Sin embargo, el olvido también se presume en otras dimensiones tales como la falta de iniciativas para su tratamiento, control o erradicación, aun en los casos en los que existen los medios necesarios para prevenir o curar esas enfermedades, muchas veces de bajo costo. En este punto, la imagen que transmite la condición de "olvidada" debe matizarse, al menos para la
Argentina. La enfermedad ha ocupado un lugar sostenido, al menos desde la década de 1950, tanto en la agenda de la política sanitaria como en el imaginario colectivo, y sigue siendo el objeto de campañas específicas para su combate, tanto por parte del gobierno como de ONG, que incluyen la lucha contra los vectores, el mejoramiento de viviendas, acciones de educación y concientización de las poblaciones donde la enfermedad sigue siendo endémica, entre otras.

El reconocimiento del Chagas como problema sanitario por parte del Estado se remonta a fines de la década de 1930. Como muestra de este interés -aún inicial- por parte del Estado, Alfredo Sordelli, Director del Instituto de Microbiología "Carlos G. Malbrán", en la X Conferencia Sanitaria Panamericana de 1938, señalaba que:

\footnotetext{
La infestación está esparcida por todo el territorio argentino [...] Desde hace poco tiempo se ha mostrado un recrudecimiento hasta el punto de que los casos llegan por lo menos a 300, de manera que consideramos el problema digno de la mayor atención (16 p.450).
}

Pero fue recién a fines de la década de 1940 cuando este reconocimiento alcanzó una importancia mayor, a partir de la confluencia de tres procesos independientes: a) la redefinición de la enfermedad en términos médicos y epidemiológicos, como una dolencia de tipo crónico y básicamente cardíaca, que afectaba a alrededor de 1.000.000 de personas en el país (los presuntos infectados con el parásito); b) la disponibilidad del gammexane como principal medio para el combate del insecto vector; y c) un contexto de política sanitaria favorable al reconocimiento y a la lucha contra las epidemias, encarnado principalmente en la figura de Ramón Carrillo, ministro de Salud del gobierno de Perón. La enfermedad de Chagas ascendió entonces a la categoría de "problema nacional" (21), y a su alrededor se comenzó a desarrollar un aparato institucional que incluyó desde programas de lucha contra el vector hasta la planificación de tareas de diagnóstico, investigación y desarrollo de alternativas de tratamiento.

Las principales políticas de intervención sobre la enfermedad estuvieron relacionadas con 
el intento de interrumpir la infección vectorial, esto es, a través de la picadura de la vinchuca. Las manifestaciones de estas políticas fueron diversas a lo largo del siglo XX. En sus inicios, fueron Ilevadas adelante por el Comité Directivo de Investigaciones y Profilaxis de la Enfermedad de Chagas, creado en 1950, la primera institución encargada oficialmente de combatir la enfermedad, que dependía del flamante Ministerio de Salud de la Nación. El Comité daba cuenta del inicio en Argentina de lo que su director, Cecilio Romaña, antiguo colaborador de Salvador Mazza, denominaba "la era de los insecticidas de acción residual y [de] la 'nueva conciencia' despertada por la medicina preventiva" (22 p.215).

A lo largo de los años, esta institución tuvo diversas transformaciones: en 1952 se convirtió en el Servicio Nacional de Profilaxis y Lucha contra la Enfermedad de Chagas, también a cargo de Romaña, órgano "encargado de la aplicación, cumplimiento y vigilancia de los programas redactados para ese fin" (Decreto 2.177 del 8 de julio de 1952). En ese marco tuvieron lugar las primeras medidas de intervención sobre la enfermedad, siguiendo las consideraciones técnicas que surgían de los trabajos experimentales de Romaña, Ilevándose a cabo las primeras campañas de rociado con insecticida (gammexane) de un modo sistemático en la provincia de Chaco, La Rioja y Catamarca, aunque estas solo tuvieron un carácter experimental y un muy corto alcance de viviendas rociadas (23).

En el período posterior al gobierno de Perón, las transformaciones institucionales y la atención a la enfermedad fueron dos constantes. La consolidación de la enfermedad de Chagas como un problema social de primer orden fue acompañada de la creación de una serie de instituciones destinadas a su identificación, medición y control de la transmisión, no solo a través de las vinchucas sino también a través de la declaración de la obligatoriedad del análisis contra la enfermedad en bancos de donación de sangre y servicios de hemoterapia (desde principios de la década de 1960), y posteriormente de la donación de órganos. Así, a partir de la segunda mitad de la década de 1950, el Servicio Nacional de Profilaxis y Lucha contra la Enfermedad de Chagas creado por el ministro Carrillo fue creciendo y transformándose hasta convertirse en el actual Instituto Nacional de Parasitología "Fatala Chabén", dedicado casi exclusivamente al diagnóstico de la enfermedad de Chagas. A esto debe sumarse la creación, a principios de la década de 1960, del Programa Nacional de Chagas, una estructura nacional dedicada a la fumigación de las viviendas rurales que durante décadas ha sido la encargada de realizar las campañas de lucha contra el vector.

Todas estas medidas han sufrido los altibajos propios de la historia argentina, y la sistematicidad en su aplicación se ha visto alterada en forma variable de acuerdo al interés que el tema haya despertado en cada una de las administraciones provinciales en cada momento histórico. Sin embargo, es posible observar una tendencia general de descenso en la transmisión de la enfermedad, por las distintas vías. Así, mientras que en el período 1964-1969 se registraba un 10\% de infectados sobre el total de la población de 18 años, a principios de la década de 1980 este porcentaje había descendido a 5,8\%, y en 1993 se registró un $1,9 \%$ de infectados en ese mismo grupo etario (24 p. 61). Actualmente, las estadísticas oficiales reflejan números similares a estos últimos, y consideran que la prevalencia media de infección por T. cruzi en niños menores de 14 años es de alrededor del 1,5\% (25).

Por cierto, estas cifras generales no reflejan la disparidad de las distintas regiones. En el caso de Argentina, mientras algunas provincias han alcanzado la certificación de la interrupción de la transmisión vectorial, existen otras donde el riesgo de transmisión sigue siendo elevado.

\section{El Chagas y la promoción de la investigación}

Además de los planes de control de la transmisión de la infección por vía vectorial y transfusional, el Chagas ha recibido una importante atención por parte de las políticas de la promoción de las investigaciones científicas, y se ha convertido así en un objeto de indagación de numerosos grupos desde variadas disciplinas científicas.

Este interés de la comunidad científica, desde fines de la década de 1960, debe entenderse a partir de una serie de factores. En primer lugar, 
una preocupación por parte de ciertos grupos de investigación por dotar a la investigación científica de relevancia social; un sostenido financiamiento del gobierno nacional para la investigación sobre el tema; la creación de un Comité de la Organización Mundial de la Salud destinado a la promoción de estas investigaciones, que funcionó como una fuente internacional de recursos desde fines de la década de 1970; y, como resultado, el desarrollo de un espacio social articulado alrededor de la enfermedad, en el que se desempeñaron diversos investigadores especialistas en Chagas en varios países de Latinoamérica, especialmente en Brasil y Argentina.

De esta forma, la investigación científica se erigió como una estrategia de intervención legítima sobre la enfermedad para los diferentes actores vinculados con la promoción, producción, circulación y difusión del conocimiento. Si bien este proceso fue amplio temáticamente, ya que la proliferación de la actividad científica sobre Chagas abarcó todas las especialidades de investigación vinculadas con la enfermedad, desde la entomología hasta la medicina y la química, la principal atención estuvo dirigida a facilitar el diagnóstico de la enfermedad y al desarroIlo, eventualmente, de una vacuna o de drogas antiparasitarias. Eso implicó que las investigaciones se concentraran mayoritariamente en dos temas: la respuesta inmune del humano ante la infección del parásito, como vía de búsqueda de una vacuna; y las características de la fisiología y el metabolismo del parásito, así como de sus reacciones ante distintas drogas.

Estas investigaciones impulsaron la generación de nuevos conocimientos sobre los distintos aspectos de la enfermedad y permitieron importantes avances en algunas áreas, principalmente en la adecuación y desarrollo de nuevos métodos de diagnóstico. Sin embargo, la apuesta política que inicialmente motorizó el apoyo a estas investigaciones no pudo verse concretada, ya que estas no llegaron a traducirse en cambios significativos en cuanto a los modos de tratamiento. De hecho, actualmente se encuentra disponible una sola droga para el tratamiento antiparasitario, el benznidazol, cuya aprobación data del año 1970 y sobre la cual se ha puesto mucho énfasis (al igual que sobre el nifurtimox, del año
1966) en la supuesta ineficacia de esos medicamentos para la etapa crónica de la enfermedad y sobre la existencia de marcados efectos secundarios sobre los pacientes.

\section{EL ENFERMO DE CHAGAS: ¿ENFERMO, INFECTADO O INVISIBLE?}

En el plano de las prácticas sociales, la enfermedad se resume en un conjunto de acciones que devienen del hecho de reconocer su especificidad. Esto es, de actores concretos que asocian a la idea del Chagas una serie de conocimientos que sirven como parámetros para sus acciones: desde los propios enfermos, los médicos que los tratan, los funcionarios, o incluso el resto de la sociedad que reacciona de una u otra manera ante la manifestación pública del problema. De esta forma, cada enfermedad constituye un rol social fundamental en la vida de los sujetos implicados: a partir del diagnóstico de la enfermedad o de la asunción de un rol específico vinculado a ella, las acciones de la vida cotidiana de los individuos implicados estarán de una u otra manera afectadas por las expectativas y los sentidos compartidos de lo que este diagnóstico implica.

En el caso del Chagas, se da una situación bastante paradójica en este aspecto, que pone de manifiesto la tensión entre visibilidad e invisibilidad que juega permanentemente en su definición como problema social. Por un lado, las estadísticas muestran una amplia población afectada, al punto que se la considera como la mayor endemia del continente en términos epidemiológicos, y solamente en Argentina se estima que hay 1.600 .000 infectados con el parásito. Pero al mismo tiempo, los sujetos directamente afectados por el Chagas no se han constituido, a lo largo de la historia de la enfermedad, como un actor colectivo significativo, capaz de establecer reclamos, reivindicaciones o derechos, como sí ha sucedido en el caso de otras enfermedades como, por ejemplo, el VIH/sida (h).

Desde el punto de vista formal, el reconocimiento de los afectados -ya sea en términos de "víctimas", de pacientes o de sujetos de derecho- ha sido una reivindicación histórica por 
parte de la comunidad médica, y ha estado implícito en cada afirmación acerca de la importancia de la enfermedad como problema sanitario, desde la incorporación de la enfermedad en los congresos especializados y su inclusión como enfermedad de denuncia obligatoria, hasta la incorporación de la pesquisa en diferentes controles sanitarios de rutina.

Pero más concretamente, a fines de la década de 1980 se sancionó una ley específica, la Ley No 22.360, que establecía que la prevención y lucha contra la enfermedad tuviera un "carácter de interés nacional", a fin de "propender al control y erradicación de esta endemia en el país" (26 Art.1). Los principales objetivos de la ley eran, por un lado, "formular las normas técnicas [...] para la elaboración, ejecución, evaluación y control de los programas de lucha", y por el otro, el "registro, orientación y tratamientos de los enfermos" (26 Art.2). El primero de estos objetivos estaba ligado a las estructuras ya existentes, y en este punto la ley sancionaba la necesidad de colaboración entre las autoridades sanitarias de los distintos distritos. En cuanto al segundo de estos objetivos, en cambio, la ley introdujo algunos cambios importantes con respecto a la situación anterior, decretando la obligatoriedad del análisis contra la enfermedad en cinco instancias centrales: en el ingreso al servicio militar; en bancos de donación de sangre y servicios de hemoterapia (ambas reglamentadas desde principios de la década de 1960); en el ingreso al nivel de escolaridad primario; en los exámenes de rutina a las mujeres embarazadas; y en los estudios preocupacionales.

En principio, esta iniciativa del Estado puede parecer favorable al reconocimiento de la enfermedad. Sin embargo, en los hechos, ha chocado con otros factores que tendieron a la "invisibilidad" de la enfermedad en el plano social. Uno de estos factores es la propia disposición a ocultar su condición por parte de los infectados con el parásito, sobre todo en instancias de búsquedas laborales, debido a la discriminación de la que pueden ser objeto. Dado que la enfermedad puede suponer una incapacidad para la realización de esfuerzos físicos, quienes se encuentran infectados por el parásito (aunque no hayan desarrollado ningún síntoma de la enfermedad) se pueden encontrar en peores condiciones para acceder a un trabajo que aquellos que no lo están (27 p.553). Paradójicamente, la ley sancionada a principios de 1980, que obligaba al examen serológico prelaboral como una forma de dar visibilidad a la enfermedad y de esa forma facilitar su control, lejos de convertirse en una herramienta útil para la incorporación de la enfermedad a la vida cotidiana de la población afectada, se transformó en un nuevo obstáculo que los individuos debieron sortear para evitar verse perjudicados, no ya por las lesiones en sí mismas, sino por las marcas que su pasado rural dejó en sus sistemas inmunológicos.

A tal punto la Ley $N^{\circ} 22.360$ tuvo esos resultados paradójicos, que esta situación fue contemplada en la sanción de la Ley 26.281 de Prevención y control del Chagas (28), en el año 2007. En esta nueva ley se recoge la declaración de la lucha y prevención del Chagas como un asunto de interés nacional, pero concentra las actividades de control en las embarazadas, los donantes de sangre u órganos, en los niños y en los recién nacidos. En cambio, en su artículo $5^{\circ}$ prohíbe la realización del examen de Chagas en los estudios prelaborales, por considerarlos discriminatorios. $\mathrm{Y}$ a tal punto ha sido fuerte la estigmatización de los infectados -el 70\% de los cuales no desarrolla ningún tipo de dolencia- que quienes más abogan por la toma de conciencia y el involucramiento de diferentes actores sociales en el combate del problema proponen que no se hable de la "enfermedad de Chagas" sino "del Chagas", constituyendo a la infección en una situación particular, pero quitándole parte de la carga simbólica negativa que implica la enfermedad (29).

Por cierto, hay otros elementos que Ilevan a la invisibilidad del Chagas, que tienen que ver con la percepción, por parte de los propios afectados, de que no se trata de una enfermedad grave (30). Según Mariana Sanmartino, esto se debe a elementos de distinto tipo: culturales, dado por la cotidianeidad con la enfermedad; y biológicos, por la larga evolución de la enfermedad -en los casos en que se desarrolla- y la falta de síntomas. A esto podríamos agregar, además, la falta de aplicación de un protocolo específico por parte de los profesionales de la salud. 


\section{REFLEXIONES FINALES}

A lo largo del artículo he intentado analizar algunas de las tensiones que atraviesan la definición del Chagas como problema sanitario, desde su concepción biomédica, las políticas orientadas a su combate, la definición de especialidades profesionales y de roles sociales asociados a la enfermedad. El propósito de esta estrategia de análisis es doble: por un lado, mostrar la complejidad que tiene la enfermedad en tanto entidad a la vez biológica, social, política, cultural, a partir de los actores concretos que la han definido como tal a lo largo de la historia. Y al mismo tiempo, lo que intenta el artículo es analizar puntualmente de qué manera, en el mismo proceso de definición de qué es lo que se entiende por la enfermedad, cómo se mide su incidencia y qué se hace para su combate, se están trazando los límites de lo que se puede hacer al respecto.

Expuesto de esta forma, más allá de las coyunturas negativas o de períodos de abandono, la persistencia del Chagas deja de ser simplemente el resultado del fracaso, del mero desinterés o de la incapacidad técnica de los medios disponibles, y se convierte en el resultado esperable y racional del accionar de los distintos actores sociales involucrados.

En este sentido, desde la perspectiva de la planificación política y de la administración de recursos, hemos mostrado que también es necesario descomponer el interés y la reacción que ha despertado la enfermedad por parte del Estado en las diferentes políticas sectoriales que impulsan iniciativas concretas dentro de su lógica de influencia. Así, mientras que en un plano discursivo la erradicación o control de la enfermedad aparece como un horizonte lejano, este objetivo debe ser luego traducido en las acciones concretas, pues esas políticas se planifican y orientan a objetivos concretos de mediano plazo, en un espacio de acción delimitado, ya sea la fumigación, el control de sangre o la promoción del conocimiento científico.

En algunos casos, la traducción entre el horizonte lejano de la erradicación y las acciones concretas que se desarrollan como consecuencia de ese interés político se choca con las limitaciones estructurales que exceden a la voluntad de los propios actores implicados. Esto es claro en el caso de la investigación científica, donde el Chagas no asume como norte la erradicación de la enfermedad sino la promoción del conocimiento asociado a ella. En esta medida, las políticas pueden reflejar resultados parcialmente exitosos, aunque luego no se den los procesos necesarios (desarrollo de nuevas drogas por parte de laboratorios, puesta a punto de nuevas técnicas para su administración masiva) para que esos conocimientos producidos puedan ayudar al objetivo último del control definitivo.

Al descomponer el discurso que supone "la lucha contra la enfermedad" en las acciones concretas, también podemos ver cómo la propia racionalidad política de administrar recursos escasos en ocasiones torna racional el abandono de las iniciativas, aunque no se haya Ilegado al objetivo máximo. En el caso de las fumigaciones, por ejemplo, el objetivo del control definitivo supone un estándar de sostenimiento de las acciones que es difícil de llevar adelante en las zonas de mayor endemia. De ese modo, la baja de los índices de infección de las últimas décadas, y el relativo éxito que esto supone, también actúa como un límite a estas iniciativas, pues a medida que la importancia del problema decrece, también es mayor la tendencia a perder el sostenimiento de las acciones y puede ser más racional destinar los recursos a otros problemas más acuciantes.

El último aspecto que intenta abordar este artículo es la consideración de las dimensiones culturales que operan en la definición de la enfermedad, tanto por parte de quienes la identifican y la nombran como de quienes la padecen. Así, aun reconociendo la relevancia social de la enfermedad, por su extensión y por el tipo de poblaciones a las que afecta, no debe perderse de vista que en el uso de esas categorías de análisis existe un ejercicio reduccionista de un discurso médico urbano y dominante que subsume una situación compleja de condiciones de vida, diversidad cultural y profundas carencias materiales a un único síntoma: la portación del parásito. La escasez de agua potable, las condiciones de precariedad de las viviendas, el acceso limitado a los servicios básicos de salud y educación, la escasez de trabajo y alimentos, el choque 
-aun- de sus culturas originarias con los modos de vida del capitalismo occidental, la falta de reconocimiento de derechos civiles, de migraciones indocumentadas, entre otros factores, quedan enmascarados detrás de un diagnóstico médico. Se trata, en definitiva, de lo que podríamos Ilamar una sinécdoque social: tomar el todo por una de sus partes, de modo tal que el Chagas se convierte en una manera de nombrar a esas poblaciones, ya sea para solidarizarse con ellas, ya sea para identificarlas como una población que solamente debe ser curada.

Este ejercicio puede realizarse de un modo más o menos crítico, ya sea destacando la imposición de un discurso médico burgués y urbano a una realidad rural extraña, ya sea valorando una estrategia de relacionamiento con distintos sectores sociales para ayudarlos a desarrollar herramientas que los ayuden a desplegar estrategias de protección y manejo de situaciones adversas. En todo caso, lo que pretende el artículo es rescatar la complejidad de la enfermedad, y recuperar la diversidad de racionalidades existentes en las perspectivas de los diferentes actores involucrados, como un modo de entender en profundidad el problema del Chagas tal como se encuentra configurado, en términos concretos, en la sociedad en la que está inmerso.

\section{NOTAS FINALES}

a. Ya sea en sus variantes optimista o fatalista (de acuerdo a la esperanza en que esos nuevos conocimientos se desarrollen), esta es la postura que subyace, en general, en las noticias relacionadas con hechos científicos o médicos que celebran un nuevo conocimiento como un "avance" contra la enfermedad, ya sea la identificación de una proteína o del genoma de un vector, aunque no sea el preponderante en el país. Como ejemplo, ver "Gran avance contra el Chagas: Iogran decodificar el genoma de la vinchuca" (4).

b. La fase crónica fue dividida por Chagas, en un primer momento, en cuatro tipos: pseudomixedematoso, mixedematoso, cardíaco y nervioso (6). La relación entre el parásito y la disfunción de la tiroides quedó reflejada en la primera denominación que tuvo la enfermedad: "Tiroiditis parasitaria".

c. El Instituto Manguinhos, liderado por Oswaldo Cruz, había sido responsable, pocos años antes del descubrimiento de Chagas, de las campañas de erradicación de la fiebre amarilla en Río de Janeiro, con el consecuente reconocimiento académico, político y civil (8).

d. Hacia 1935 los casos en los que se habían comprobado microscópicamente la existencia del parásito en humanos no llegaban a 50, ver Yorke (11) citado en Kropf (9 p.246). e. El primer cuestionamiento a la relación entre parásito y bocio provino de los estudios de Rudolph Kraus en el Instituto Bacteriológico del Departamento Nacional de Higiene (12).

f. Los trabajos de Mazza incluyeron la identificación de los primeros casos a mediados de la década de 1920, la comprobación del parásito en el territorio y de la existencia de vectores, el análisis de los reservorios naturales del parásito, la identificación de casos agudos y crónicos, la descripción de las características de cada etapa, el análisis de los métodos de diagnóstico y finalmente el ensayo de tratamientos (estos últimos sin éxito) (15).

g. Los trabajos de Laranja y Nóbrega en Brasil, pertenecientes al Centro de Pesquisas de Bambuí, y de Rosenbaum y Álvarez, pertenecientes al Hospital Ramos Mejía, fueron fundamentales en la definición de la "miocardiopatía chagásica crónica" (13).

h. Ante situaciones de escasez de medicamentos, o siquiera la amenaza de la escasez, las diferencias entre estos dos colectivos son notables. Mientras que los afectados por el VIH/sida han realizado movilizaciones exigiendo la garantía en la provisión de las drogas, los afectados por el Chagas no se han convertido en un actor público de peso ante las noticias de que la droga no estaría más disponible. 


\section{REFERENCIAS BIBLIOGRÁFICAS}

1. Kropf SP. Doença de Chagas, doença do Brasil: ciência, saúde e nação (1909-1962). Rio de Janeiro: Editora Fiocruz; 2009.

2. Delaporte F. La maladie de Chagas: histoire d'un fléau continental. Paris: Payot et Rivages; 1999.

3. Organización Panamericana de la Salud, Organización Mundial de la Salud. Estrategia y plan de acción para la prevención, el control y la atención de la enfermedad de Chagas (CD50/16) [Internet]. 9 ago 2010 [citado 18 jul 2012]. Disponible en: http://new.paho.org/hq/dmdocuments/2010/CD50-16-s.pdf

4. Gran avance contra el Chagas: logran decodificar el genoma de la vinchuca. Clarín [Internet]. 17 may 2012 [citado 18 jul 2012]. Disponible en: http://www.clarin.com/sociedad/Gran-Chagasdecodificar-genoma-vinchuca_0_701929898 .html

5. Rosenberg C, Golden J. Framing disease: Studies in cultural history. New Jersey: Rutger University Press; 1992.

6. Chagas C. Nova entidade morbida do homem. Brazil-Medico. 1910;24(27):263-265; (43):423428; (44):433-437; (45):443-447.

7. Chagas C. Nova tripanozomiase humana: Estudos sobre a morfolojia e o ciclo evolutivo do Schizotrypanum cruzi, ajente etiolojico de nova entidade morbida do homem. Memorias do Instituto Oswaldo Cruz. 1909;1(2):159-218.

8. Benchimol J, Teixeira L. Cobras e lagartos \& outros bichos: Uma história comparativa dos institutos Butantã e Oswaldo Cruz. Rio de Janeiro: Fiocruz, Editora UFRJ; 1993.

9. Kropf SP. Doença de Chagas, doença do Brasil: ciência, saúde e nação (1909-1962) [Internet]. [Tese de Doctorado em Historia]. Niteroi: Universidade Federal Fluminense; 2006 [citado 18 jul 2012]. Disponible en: http://ged1.capes.gov.br/ CapesProcessos/926738-ARQ/926738_6.PDF

10. Hochman G. Brasil no es solo enfermedad: Juscelino Kubitschek y la búsqueda de una nueva representación para el país. En: Hochman G, Di Liscia MS, Palmer S, organizadores. Patologías de la Patria: enfermedades, enfermos y nación en América Latina. Buenos Aires: Lugar Editorial; 2012.
11. Yorke W. Chagas' disease: A critical review. Tropical Diseases Bulletin. 1937;34(4):275-300.

12. Kraus R, Maggio C, Rosenbusch F. Bocio, cretinismo y enfermedad de Chagas: Segunda comunicación. La Prensa Médica Argentina. 1916;III(17):177-180.

13. Zabala JP. La enfermedad de Chagas en Argentina: investigación científica, problemas sociales y políticas sanitarias. Bernal: Universidad Nacional de Quilmes; 2010.

14. Sierra-Iglesias JPS. Salvador Mazza, redescubridor de la enfermedad de Chagas: Su vida, su obra. San Salvador de Jujuy: Universidad Nacional de Jujuy; 1990.

15. Mazza S. La enfermedad de Chagas en la República Argentina. En: Actas y Trabajos del VI Congreso Nacional de Medicina, t. 3. Rosario: Editorial Pomponio; 1939. p. 9-20.

16. Sordelli A. Tripanosomiasis americana. En: Actas de la Décima Conferencia Sanitaria Panamericana (Publicación No 136). Bogotá: Oficina Sanitaria Panamericana; 1939. p. 450.

17. Dias E. O signal de Romaña na molestia de Chagas. Acta Médica. 1939;3(4):60-62.

18. Mazza S. Sobre el desacierto de adoptar un subtítulo para la enfermedad de Chagas. La Prensa Médica. 1943;30(10):433-440.

19. Chagas C. Estado atual da tripanossomíase americana. Revista de Biologia e Higiene. 1934;5(2):58-64.

20. Kreimer P, Romero LA, Bilder P. ¿Tratar o no tratar?: La controversia científica sobre el tratamiento para los enfermos de Chagas crónico. Asclepio. 2010;62(2):405-428.

21. Zabala JP. La enfermedad de Chagas como "problema social nacional" en Argentina: Populismo, sanitarismo e investigación científica. En: Hochman G, Di Liscia MS, Palmer S, organizadores. Patologías de la Patria: enfermedades, enfermos y nación en América Latina. Buenos Aires: Lugar Editorial; 2012.

22. Romaña C. Panorama continental de la enfermedad de Chagas. En: Anais do IX Congresso Brasileiro de Higiene. Porto Alegre; 1951. p. 209-215.

23. Silveira AC. Situação do controle da transmissão vetorial da doença de Chagas nas Américas. Cadernos de Saúde Pública. 2000;16(Supl. 2):S35-S42. 
24. Segura E. El control de la enfermedad de Chagas en la República Argentina. En: Silveira A, editor. El control de la enfermedad de Chagas en los países del Cono Sur de América: Historia de una iniciativa internacional. Buenos Aires: Organización Panamericana de la Salud; 2002.

25. Programa Nacional de Chagas. El Chagas en el país y América Latina [Internet]. Buenos Aires: Ministerio de Salud [citado 18 jul 2012]. Disponible en: http://www.msal.gov.ar/chagas/ index.php/informacion-para-ciudadanos/el-chagasen-el-pais-y-america-latina.

26. Argentina. Ley 22.360 de Lucha contra el Mal de Chagas [Internet]. 31 dic 1980 [citado 18 jul 2012]. Disponible en: http://www.alcha.org.ar/ Articulos/Ley\%2022360.pdf
27. Storino R, Milei J. Aspectos legales y laborales. En: La enfermedad de Chagas. Buenos Aires: Editorial Doyma; 1994.

28. Argentina. Ley 26.281 de Prevención y Control del Chagas [Internet]. 8 ago 2007 [citado 18 jul 2012]. Disponible en: http://www.msal. gov.ar/chagas/index.php/institucional/ley-26281

29. Sanmartino M. ¿Qué es lo primero que piensa cuando escucha la palabra "Chagas"? Revista de Salud Pública. 2009;(XIII)1:74-78.

30. Sanmartino M. Faire face à la maladie de Chagas en partant des conceptions des populations concernées. [Tesis de doctorado]. Genève: Faculté de Psychologie et des Sciences de l'Éducation, Université de Genève; 2006.

\section{FORMA DE CITAR}

Zabala JP. La enfermedad en su laberinto: avances, desafíos y paradojas de cien años del Chagas en Argentina. Salud Colectiva. 2012;8(Supl 1):S9-S21.

Recibido el 10 de julio de 2012

Aprobado el 21 de agosto de 2012 


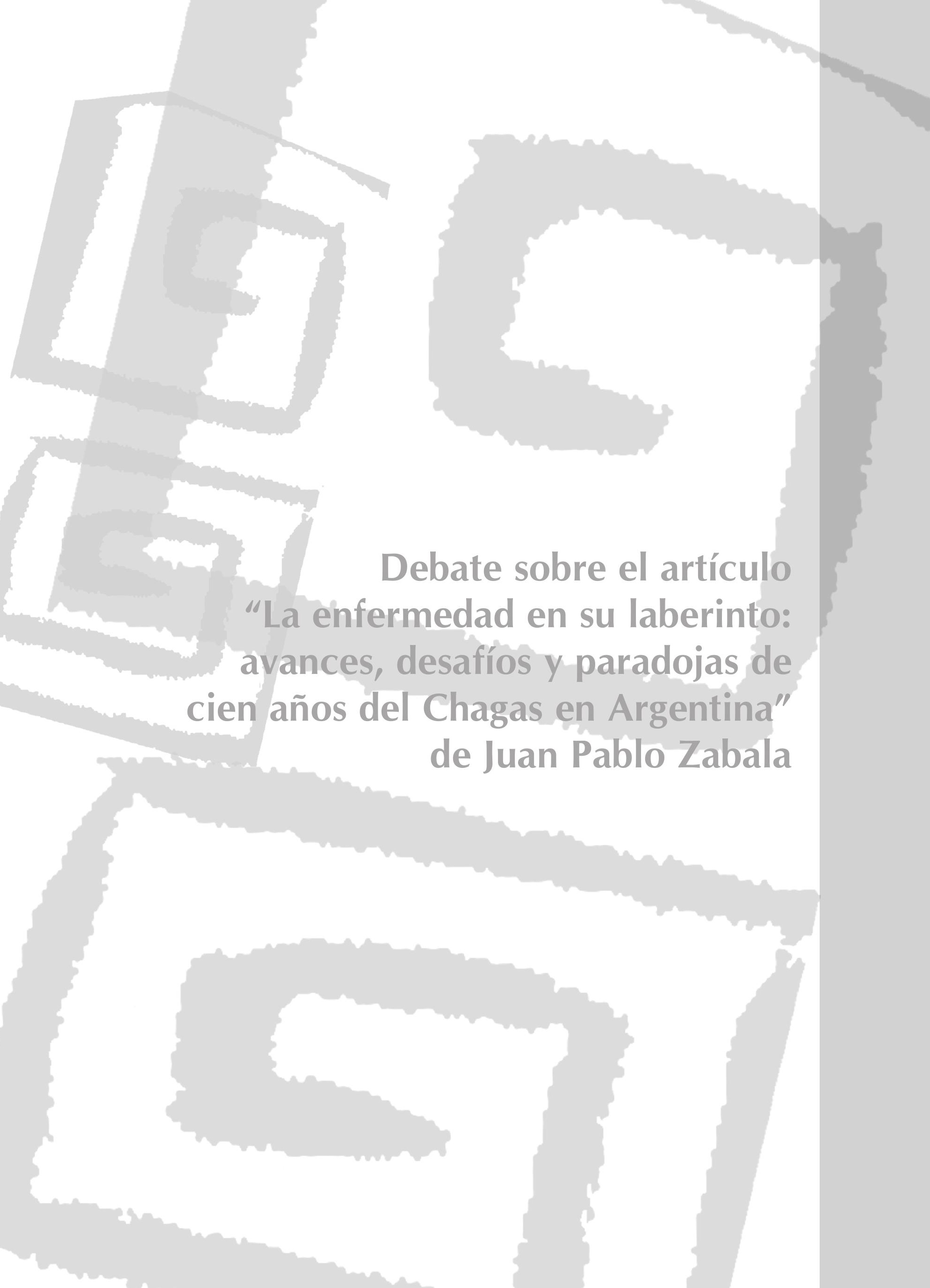

